\title{
High-Precision Ranging and Range-Rate Measurements over Free-Space-Laser Communication Link
}

\author{
Guangning Yang, Wei Lu, Michael Krainak, Xiaoli Sun \\ NASA Goddard Space Flight Center (GSFC) \\ Greenbelt, MD \\ Guangning.yang-1@nasa.gov
}

\begin{abstract}
We present a high-precision ranging and range-rate measurement system via an optical-ranging or combined ranging-communication link. A complete bench-top optical communication system was built. It included a ground terminal and a space terminal. Ranging and range rate tests were conducted in two configurations. In the communication configuration with 622 data rate, we achieved a two-way rangerate error of $2 \mu \mathrm{m} / \mathrm{s}$, or a modified Allan deviation of $9 \times 10^{-15}$ with 10 second averaging time. Ranging and range-rate as a function of Bit Error Rate of the communication link is reported. They are not sensitive to the link error rate. In the single-frequency amplitude modulation mode, we report a two-way range rate error of $0.8 \mu \mathrm{m} / \mathrm{s}$, or a modified Allan deviation of $2.6 \times 10^{-15}$ with 10 second averaging time. We identified the major noise sources in the current system as the transmitter modulation injected noise and receiver electronics generated noise. A new improved system will be constructed to further improve the system performance for both operating modes.
\end{abstract}

Index Terms-Space Optical ranging, Doppler range rate, Doppler ranging, space optical navigation, space optical communication, formation fly.

\section{Table of Contents}

1. INTRODUCTION . .1

2. CONFIGURATION AND PERFORMANCE ...................... 2

3. DISCUSSION

4. CONCLUSION

ACKNOWLEDGMENT ..................................................... 12

REFERENCES............................................................. 12

BIOGRAPHY ...................................................................... 12

\section{INTRODUCTION}

In spacecraft navigation and tracking, range and range-rate are the most commonly used tracking observables. Currently this is done with Doppler shift of a microwave carrier that provides the spacecraft-Earth relative velocity along the line of sight $[1,5]$. ESA and NASA Doppler tracking systems rely on two-way coherent radio links at X-band (7.2-8.4 GHz). The average measurement accuracy ranges from 100 to $20 \mu \mathrm{m} / \mathrm{s}$ at 60 s integration time. ESA has conducted a study for enhancement of end-to-end accuracy for spacecraft tracking techniques. It sets the target accuracies to $10 \mu \mathrm{m} / \mathrm{s}$ at $60 \mathrm{~s}$ integration time for Doppler (two-way) [6].

High-precision Doppler ranging also enables the gravitational science missions such as Gravity Recovery and Climate Experiment (GRACE [7]) and Gravity Recovery and Interior Laboratory (GRAIL). GRAIL is a spacecraft-tospacecraft tracking mission that was developed to map the structure of the lunar interior by producing a detailed map of the gravity field [4].

One of the primary noise sources with RF Doppler ranging is the plasma (interplanetary and Earth ionosphere). It becomes a performance limitation when Sun-Earth-probe (or SEP angle) is less than 30 degrees [2]. This noise source impacts both interplanetary and low-earth-orbit (LEO) Doppler tacking accuracies. To achieve the NASA and ESA required performance specifications, a dual frequency RF ranging system was needed to calibrate out the plasma caused ranging error [5]. This increased the instrument size and complexity.

To overcome the plasma limit to the signal-to-noise ratio of $\mathrm{RF}$ ranging, the ranging measurement can be perform on an optical carrier with a RF subcarrier or with synchronized optical communication links. The Lunar Laser Communication Demonstration (LLCD) has conducted such synchronized laser ranging tests over an optical communication link. LLCD achieved sub-centimeter ranging accuracy [3]

There are several advantages of ranging with the optical carrier. First, plasma scintillation phase noise [5] is one of the fundamental performance noise sources for RF ranging, especially at small Sun-Earth-Probe (SEP) angles $[1,5]$. The magnitude of this noise is proportional to square of the wavelength [5]. The optical carrier has a significantly shorter wavelength. This eliminates the effects of the plasma on the noise in optical ranging. Second, the high directionality of the optical signal (high antenna gain) makes long ranging links feasible. Third, the directionality of optical beam makes optical ranging instrument very small. It makes a Cubesat-compatible high-precision science instrument feasible. Fourth, the clock signal is intrinsic for synchronized optical communication. There is no major hardware upgrade on an optical communication platform with clock recovery hardware already present. 
This paper is arranged as follows: First, an introduction gives an overview of our work and the related background. Second, the instrument configurations and corresponding test results are presented. It includes a description of a Dual Mixer Time Difference (DMTD) measurement apparatus. The DMTD provides increased system sensitivity. We also give a description of two ranging operating modes and results: a) laser communication mode, b) single-tone optical amplitude modulation (AM) mode. Third, we show our data analysis and give a performance comparison of the different ranging modes, noise sources, and predicted instrument sensitivity. Last, a technical summary will be presented.

\section{Configuration and Performance}

The ranging measurements were conducted in two different modes: ranging over optical communication (Fig. 1), where the recovered data clock is used for ranging and range rate measurement, and ranging over single tone optical amplitude modulation (Fig. 2), where the detected RF tone is used for ranging and range-rate measurements. All of clock phase measurements were conducted with the DMTD test apparatus.

We were using as many commercial-off-the-shelf (COTS) parts as possible to lower the cost and shorten the development time. The OC-12 protocol parts are selected for its availability, which has a data rate of $622 \mathrm{Mbps}$.

\section{DMTD test apparatus and performance}

All measurements of the frequency stability measurements used the DMTD. The best time-frequency counter on the market has time timing accuracy of $\sim 10 \mathrm{ps}$, which will have an instrument sensitivity level at $10^{-11}$ for relative accuracy at 1 second averaging time. We require a sensitivity level of better than $10^{-14}$. A Dual Mixer Time Difference (DMTD) clock measuring system (see Fig. 3) was constructed. A DMTD system is a well-established way to make high-resolution phase measurements on precision frequency sources [10]. Both "Clock under Test" and "Ref Clk" are mixed with an RF signal ( $\Delta$ Frequency Oscillator). The $\Delta$ Frequency Oscillator has a small frequency offset from "Clock under Test" and "Ref Clk" to generate IF frequencies as the output of the mixer. Two IF signals at the output of the mixers are low pass filtered and amplified before the "Time Interval Counter". The signal phase is measured as the zero-crossing time difference of the two IF signals.

For the instrument noise floor test (see Fig. 4), a single RF source at $622 \mathrm{MHz}$ is divided into two and feeds both Sig-Port and Ref-Port. The $\Delta$ Fre Oscillator frequency is set at 622.001MHz, which gives a $1 \mathrm{KHz}$ IF frequency. The heterodyne mixing gain for signal phase measurement is $3.11 \times 10^{5}(622 \mathrm{MHz} / 1 \mathrm{kHz})$, which increases the counter sensitivity by 5 orders of magnitude.

The phase data and Allan Deviation of the system are shown in Fig. 5 and Fig. 6 respectively. Fig. 5 is a phase error plot with a sampling rate of $1 \mathrm{KHz}$. It has a zero-to-peak value of $\sim 50 \mathrm{fs}$. As shown in Fig. 6, the system has achieved a noise floor of $1.6 \times 10^{-14}$ for the Allan deviation and $9.3 \times 10^{-16}$ for the modified Allan deviation at a 1 second averaging time.

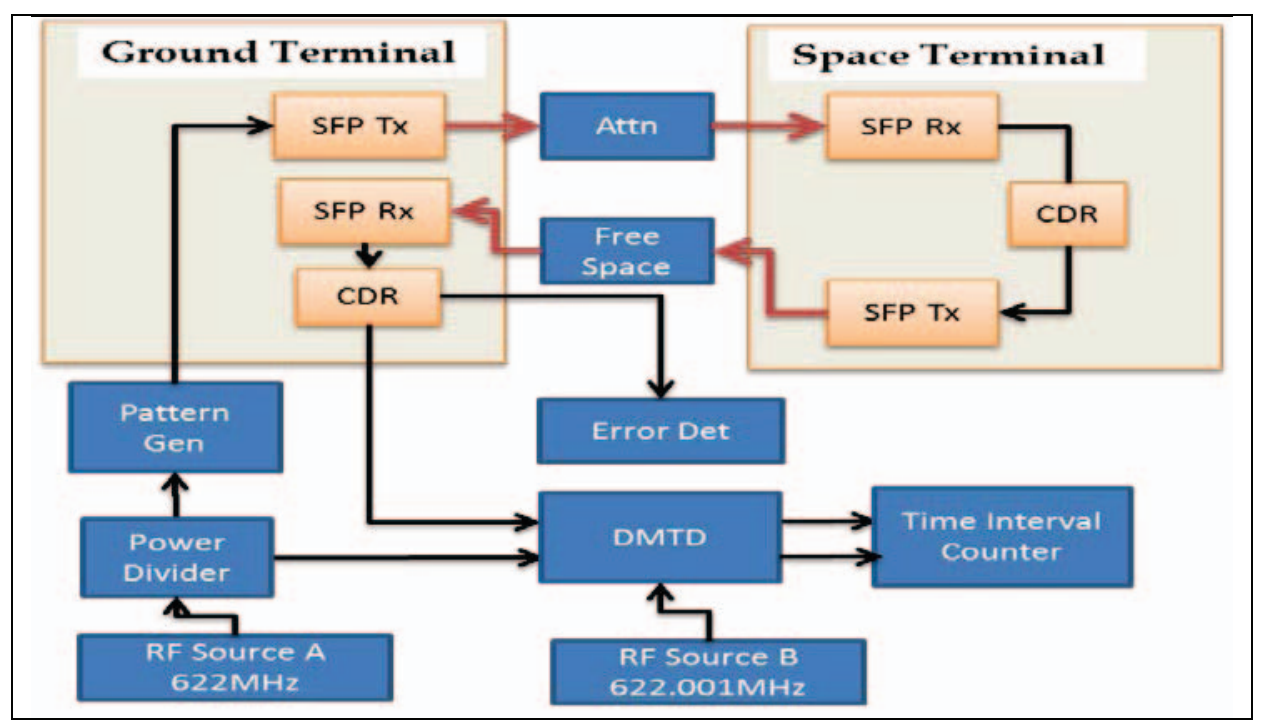

Figure 1. Ranging over optical communication configuration. "SFP Tx"( Telecom OC-12 optical transmitter), "SFP Rx" (Telecom OC-12 optical receiver,"), "CDR" (clock data recovery circuit), "Attn" (variable optical attenuator), "Free Space"( Optical free space coupling telescope), "Pattern Gen" (communication data pattern generator), "Error Detector" (communication error detector), "Power Divider" (3 dB RF power divider), "RF Source" (low noise RF frequency synthesizer), "DMTD" (Dual Mixer Time Difference measurement system), "Time Interval Counter" (high precision frequency and time interval counter). 


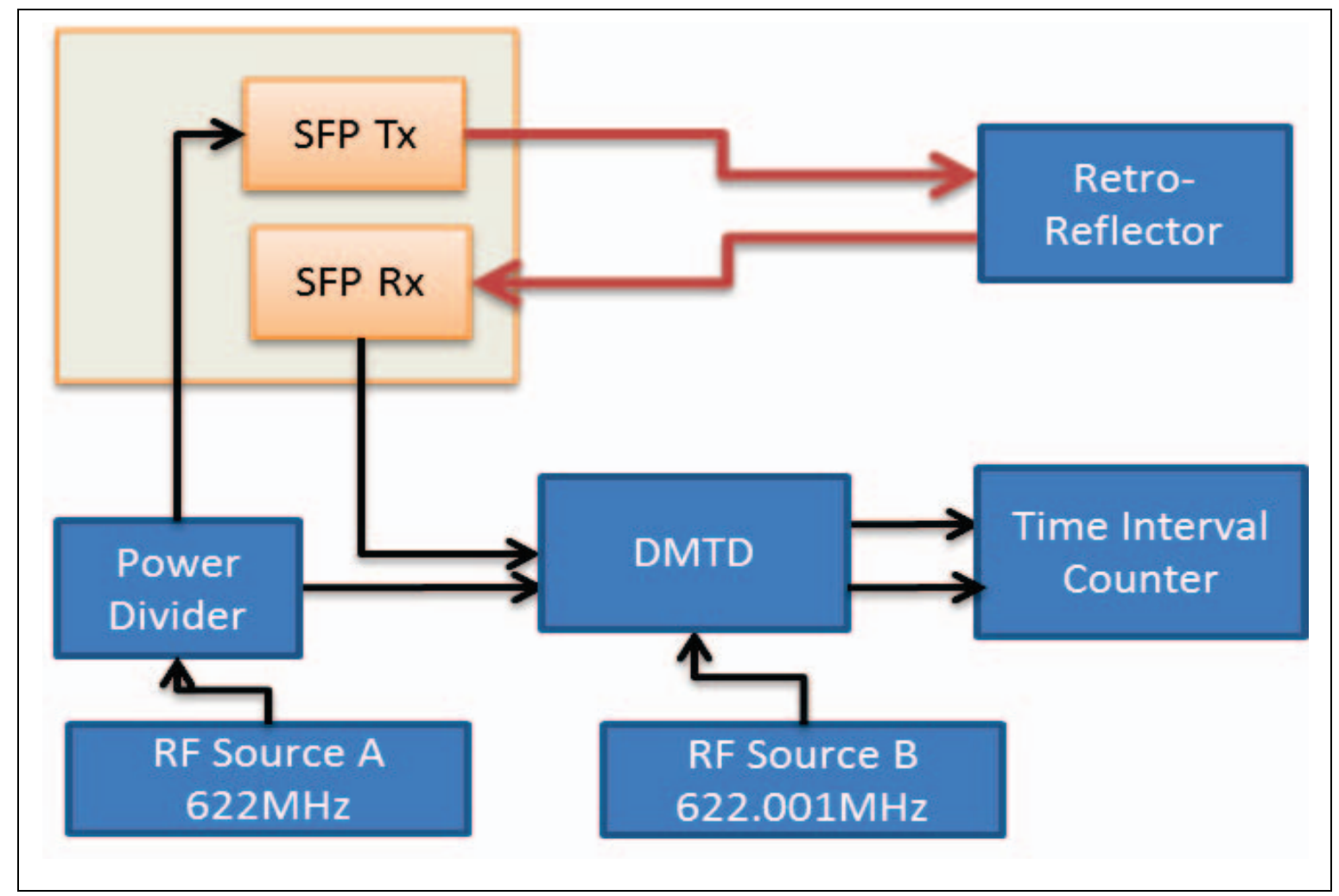

Figure 2. RF ranging over optical tone modulation configuration. "SFP Tx"( Telecom OC-12 optical transmitter), "SFP Rx" (Telecom OC-12 optical receiver,"), "Retro-Reflector" (optical retro-reflector) "Power Divider" (3 dB RF power divider), "RF Source" (low noise RF frequency synthesizer), "DMTD” (dual mixer time difference measurement system), "Time Interval Counter" (high precision frequency and time interval counter).

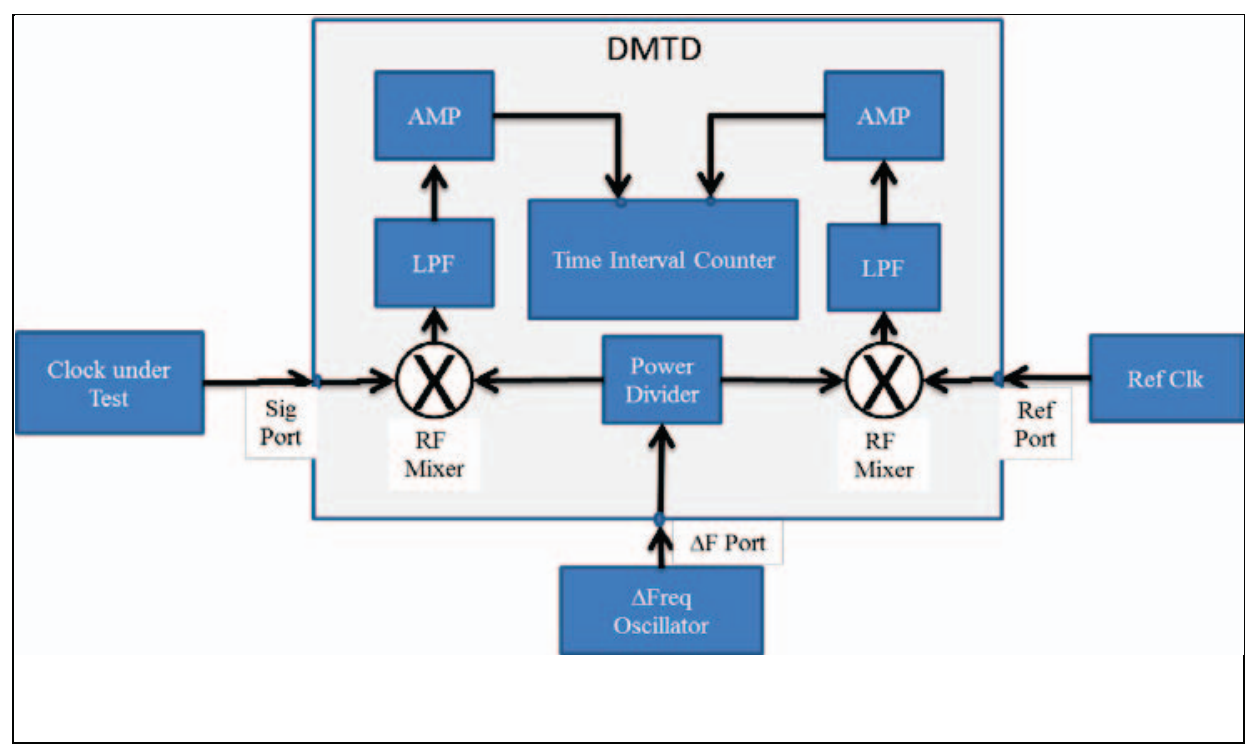

Figure 3. Dual Mixer Time Difference (DMTD) phase measurement setup. “AMP” (low frequency amplifier for IF frequency) 


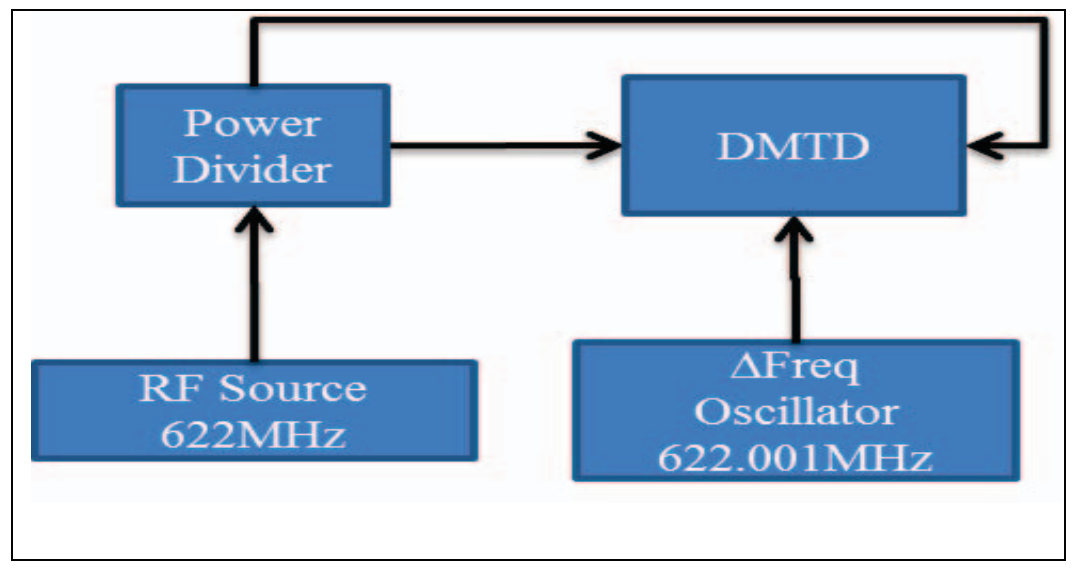

Figure 4. Dual Mixer Time Difference (DMTD) noise floor test configuration. A frequency offset of $1 \mathrm{KHz}$ is used for IF frequency after mixer.

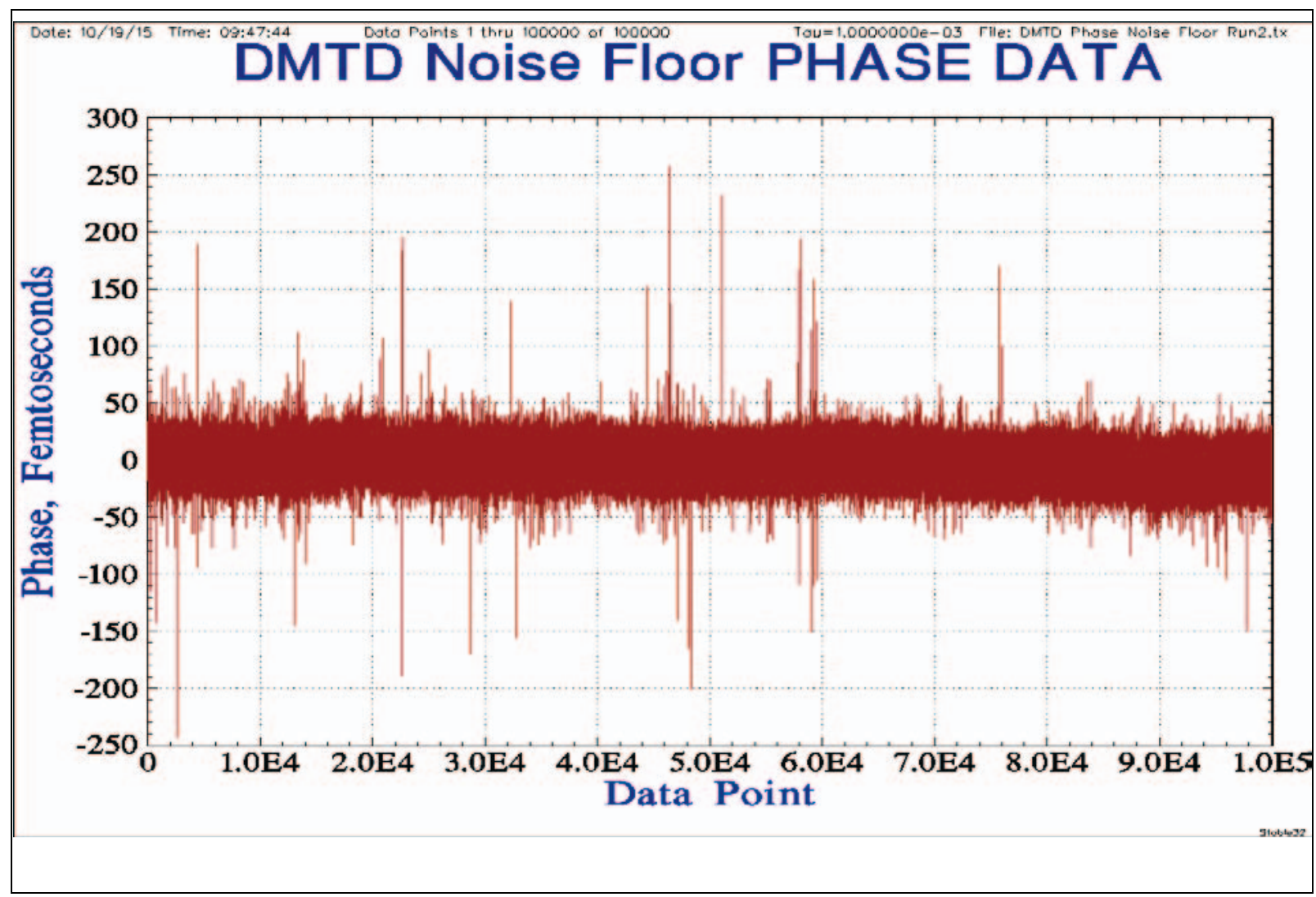

Figure 5. Dual Mixer Time Difference (DMTD) phase measurement noise floor test results. Sequential phase error with sampling rate of $1 \mathrm{KHz}$ 


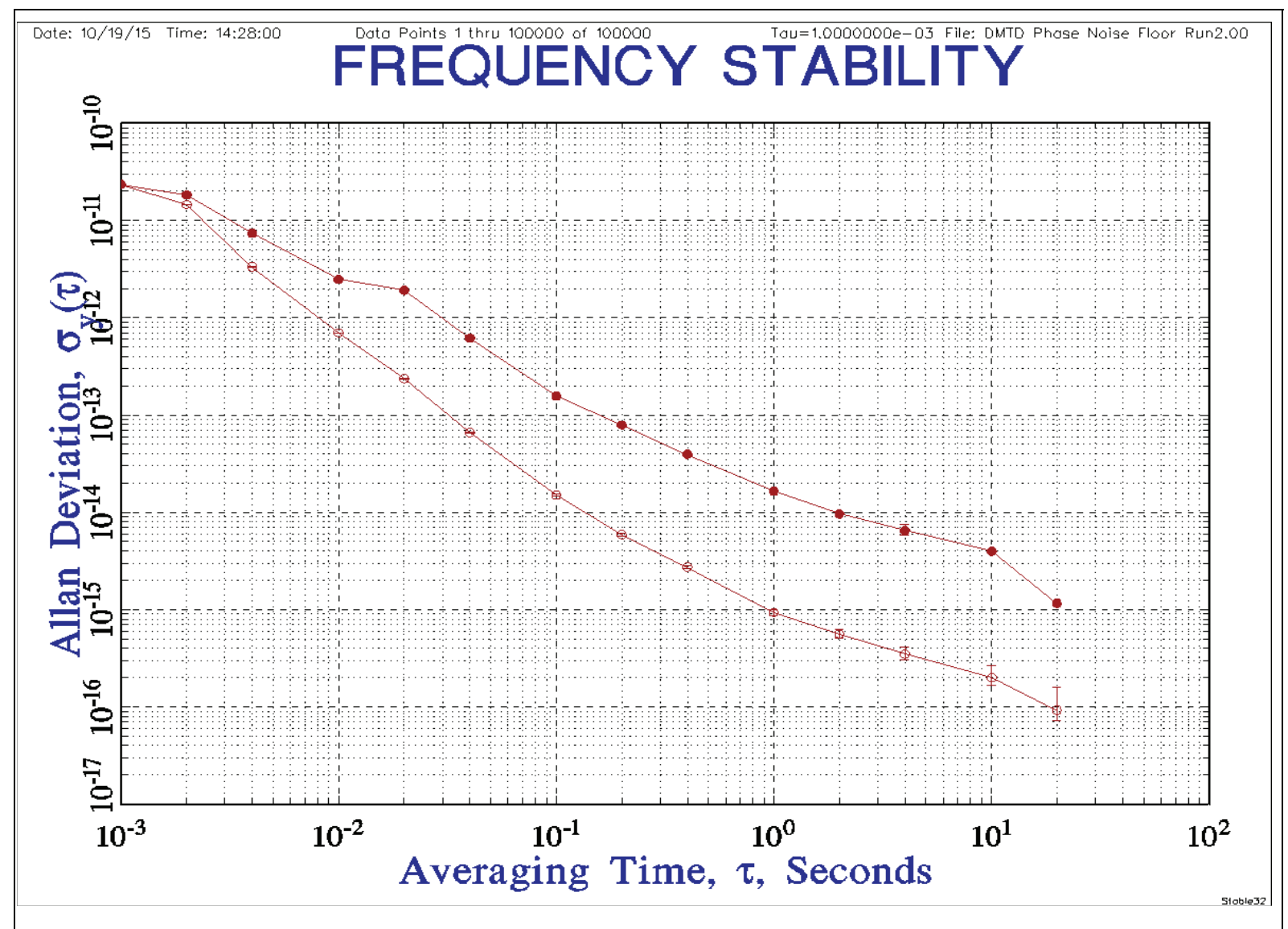

Figure 6. Dual Mixer Time Difference (DMTD) phase measurement noise floor test results. Allan and Modified Allan deviation as a function of averaging time.

\section{Optical communication mode ranging performance}

The ranging over optical communication link test configuration is shown in Fig.1. The system is operating at 622 Mbps (OC-12 rate) with a pseudo-random bit sequence (PRBS) $2^{31}-1$. The recovered clock is used for range-rate measurements.

The signal flow (Fig. 1) is: "RF Source A" generates an RF clock at $622 \mathrm{MHz}$. It is divided into two signals with a "Power Divider". One power divider output is used as DMTD reference input for the clock phase measurement. The other output of is driven the pattern generator to generate a PRBS $2^{31}-1$ pattern at $622 \mathrm{Mbps}$, followed by a laser transmitter ("SFP Tx", a commercial OC-12 transmitter with wavelength of $1550 \mathrm{~nm}$ ). The optical output from the ground transmitter is then connected to the space receiver through an optical attenuator. The space terminal receives the signal, and realigns the data with the clock via the clock data recovery circuit. The realigned data is then looped back to drive the space optical transmitter. Then the optical signal is free-space coupled to the ground optical receiver. The signal is fed to the ground clock data recovery (CDR) circuit, one clock and one data signal are fed to the error detector for bit error rate testing. The other clock signal is fed the Signal Port of the DMTD for clock phase measurement. A second RF source is generating an RF signal at $622.001 \mathrm{MHz}$ to drive the DMTD $\Delta \mathrm{F}$ port.

The range-rate accuracy as a function of BER is tested by varying receiver optical power.

All phase measurements of the recovered clock signal are taken with DMTD with sampling rate of $1 \mathrm{KHz}$. A typical phase measurement plot is shown in Fig.7. The standard deviation of the phase amplitude is 0.5 ps, giving a 3 -sigma value of $1.5 \mathrm{ps}$.

Fig. 8 is the modified Allan deviations vs. averaging time for different receiver optical powers. The Allan deviations are not changing for all receiver optical powers $(-18 \mathrm{dBm}$ to $33 \mathrm{dBm}$ ) until the receiver CDR has a "Loss of Lock" at "$33.17 \mathrm{dBm}$ " receiver power level. Under the "Loss of Lock" condition, the CDR output clock is not locked to the received data clock. At that point, the corresponding Allan deviation is no longer valid (Fig. 8, Trace “-33.17 dBm”).

Fig. 9 is a plot of range-rate accuracy as a function of BER for averaging time of 1 second and 10 seconds. Again, the range-rate accuracies are the same for the entire BER rates until the CDR has the "loss of lock". Range rate accuracies of $25 \mu \mathrm{m} / \mathrm{s}$ for 1 second averaging time and $2.1 \mu \mathrm{m} / \mathrm{s}$ for 10 second averaging time were achieved. 


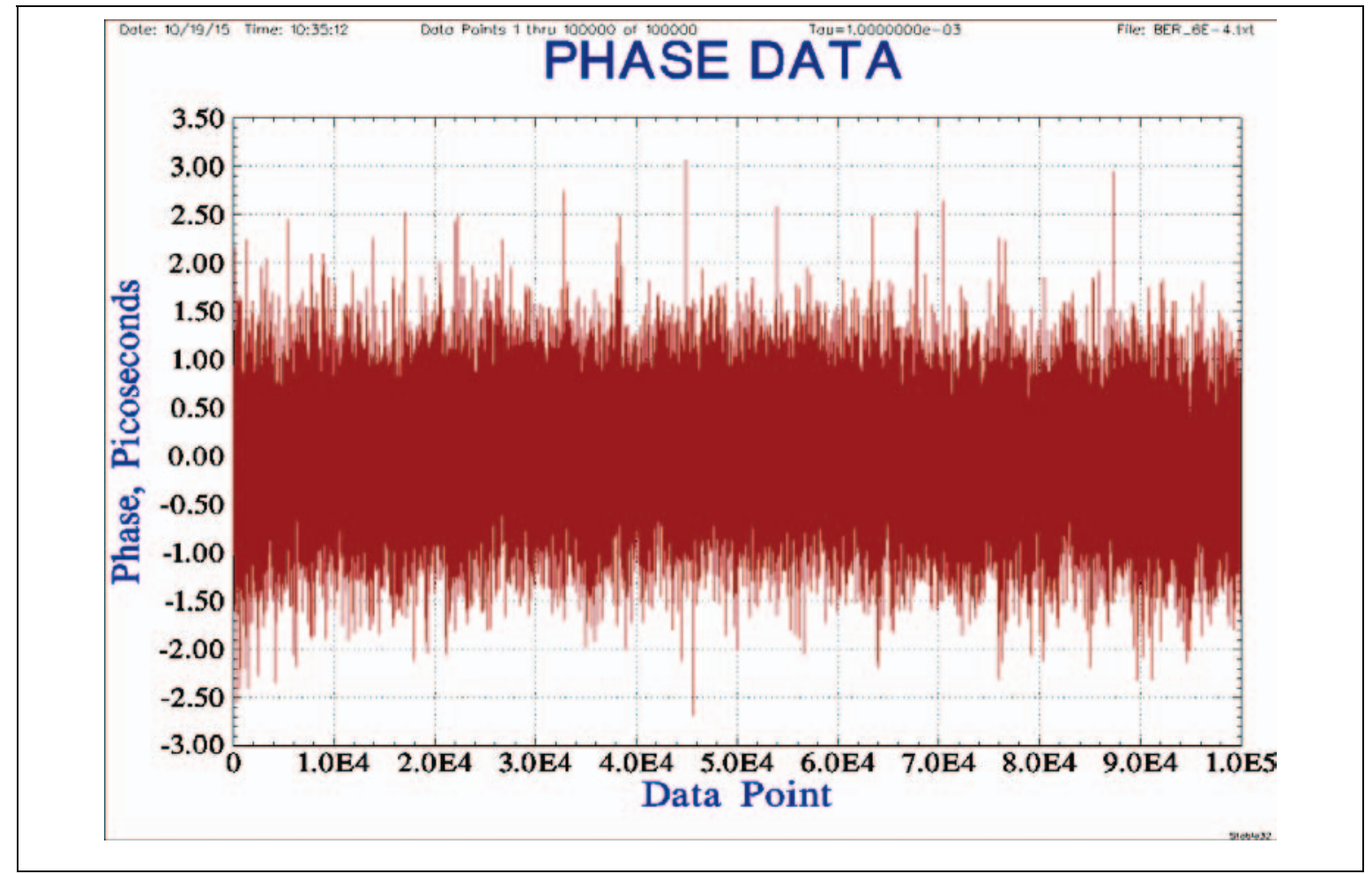

Figure 7. RF ranging over 622MBPS optical communication: Phase measurements with an averaging time of 0.001s for a $B E R=6 \times 10-4$ data link performance.

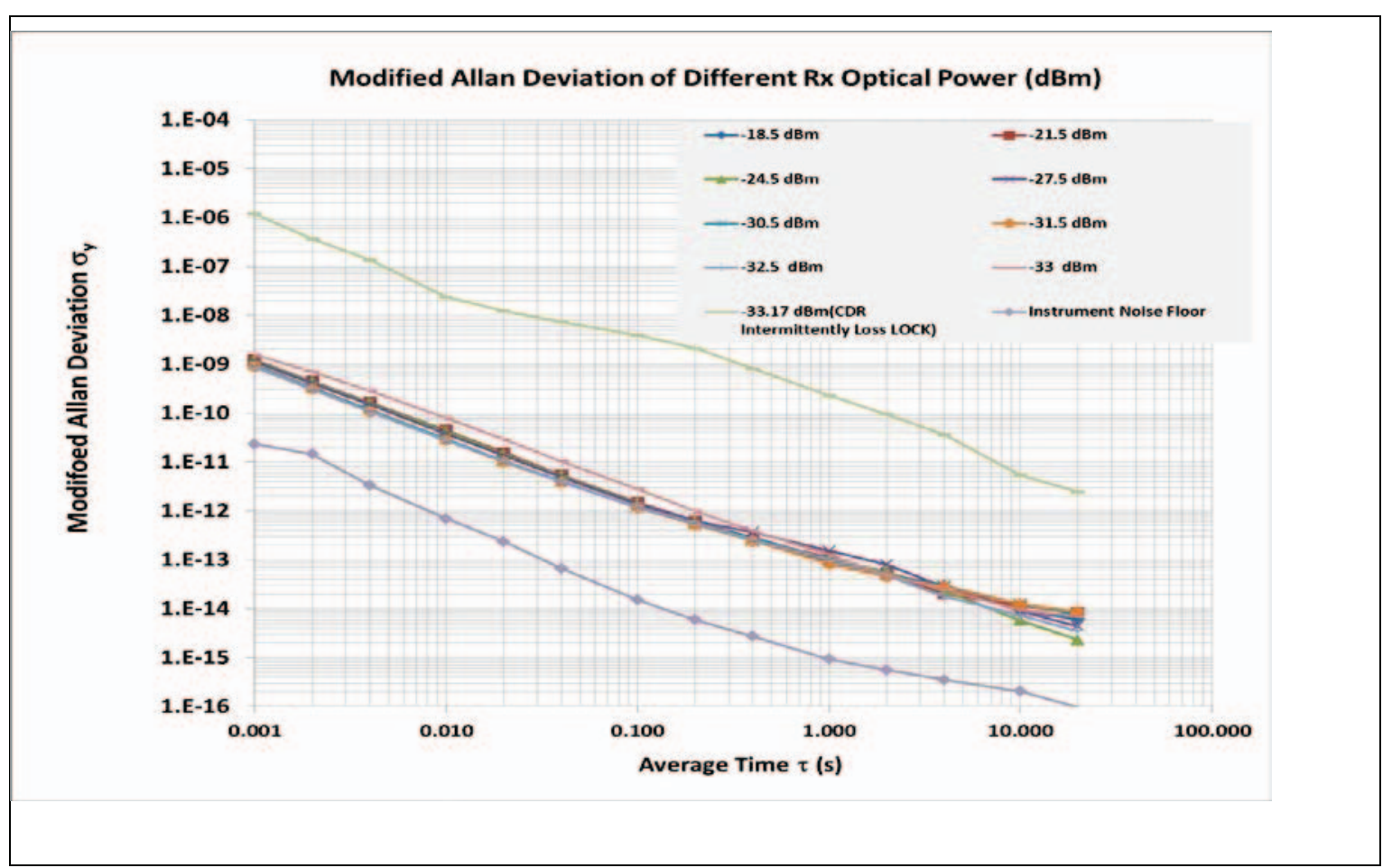

Figure 8. Modified Allan deviation of RF ranging over 622MBPS optical link for different receiver optical power. Instrument noise floor is also plotted for reference. $\mathrm{X}$-axis is the averaging time of the frequency measurement, $\mathrm{y}$-axis is the Modified Allan deviation 


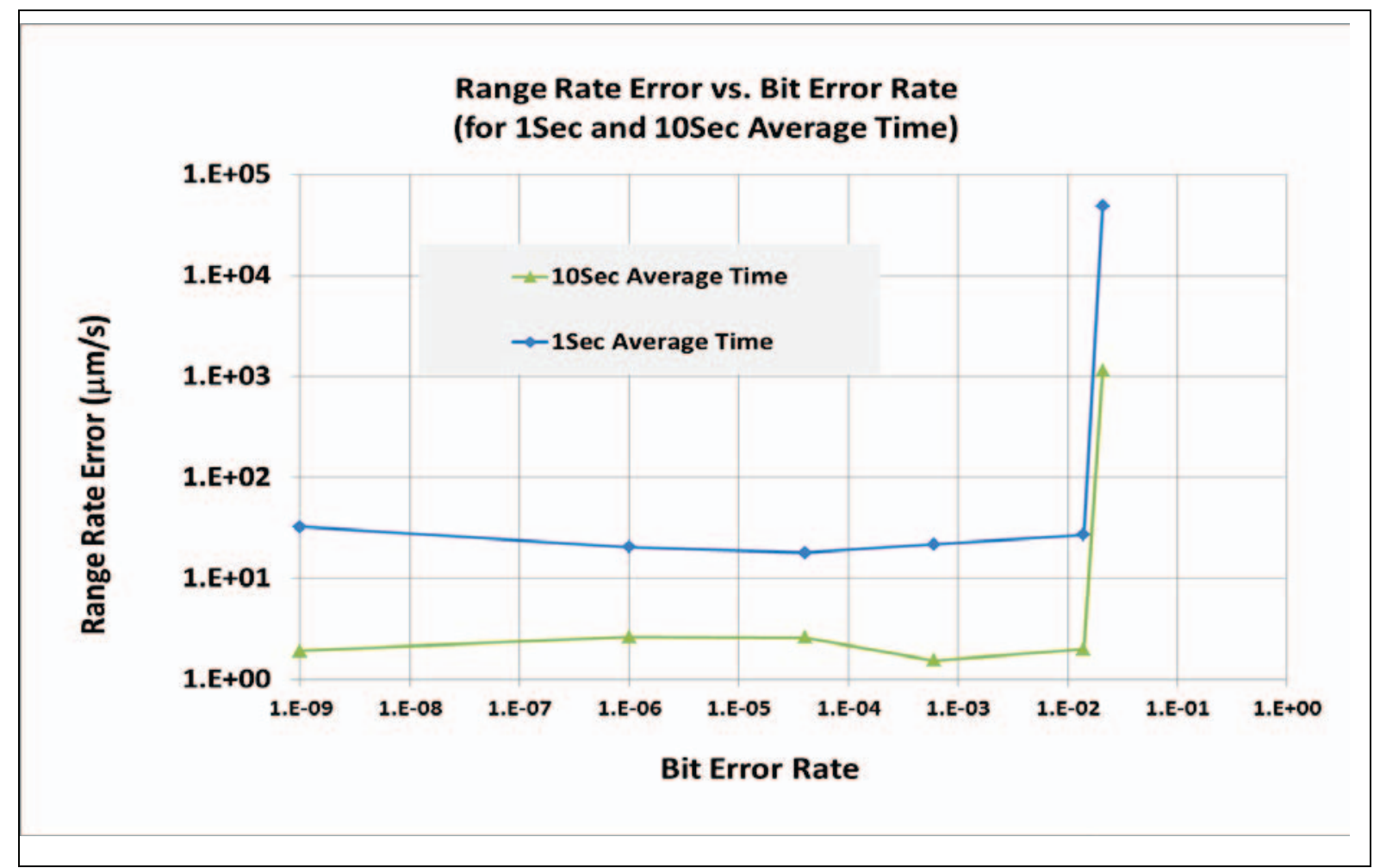

Figure 9. Range-rate error as a function of receiver optical power. $X$-axis is the bit error rate (BER) of the communication link, Y-axis is the range rate error in $\mathbf{u m} / \mathbf{s}$. Two traces are shown in the figure for different average time of the frequency measurement (1second and 10 second).

\section{Single tone mode ranging performance}

Ranging measurements were also conducted with single tone intensity modulation. Compared to the optical communication clock signal, the single-tone AM modulation will provide improved $622 \mathrm{MHz}$ RF signal power since there is no data modulation to introduce spectral broadening. This improves the signal-to-noise ratio for the clock used in the ranging measurement.

Similar to the optical communication ranging configuration, the signal flow (Fig. 2) is: "RF Source A" generates an RF clock at $622 \mathrm{MHz}$. It is divided into two signals with a "Power Divider". One power divider output is used as DMTD reference input for clock phase measurement. The other output drives a laser transmitter. The optical output is reflected back to a receiver by a retro-reflector. The receiver RF signal is fed to the Signal Port of the DMTD for clock phase measurement. A second RF source is generating an RF signal at $622.001 \mathrm{MHz}$ to drive the DMTD $\Delta \mathrm{F}$ port.

Fig. 10 shows the phase measurements of the $622 \mathrm{MHz}$ tone. It has a zero-to-peak value of $300 \mathrm{fs}$ with an averaging time of $0.001 \mathrm{~s}$. Fig. 11 gives the Allan and modified Allan deviation. Overall, it is factor of 6 away from the instrument noise floor. However, compared to the ranging over communication in Fig. 8. There is a factor of 10 improvements. The main contribution of the improvement is the increased RF signal power at $622 \mathrm{MHz}$ frequency. There is flicker FM noise for averaging times in range of $0.1 \mathrm{~s}$ to $1 \mathrm{~s}$. This flicker noise limits the range-rate accuracy for $1 \mathrm{~s}$ averaging time at 19.7 $\mu \mathrm{m} / \mathrm{s}$. The signature of this flicker noise is also visible from the phase measurement plot in Fig.10. The range-rate accuracies are $19.7 \mu \mathrm{m} / \mathrm{s}$ and $0.27 \mu \mathrm{m} / \mathrm{s}$ for a 1 second and 10 second averaging time respectively. 


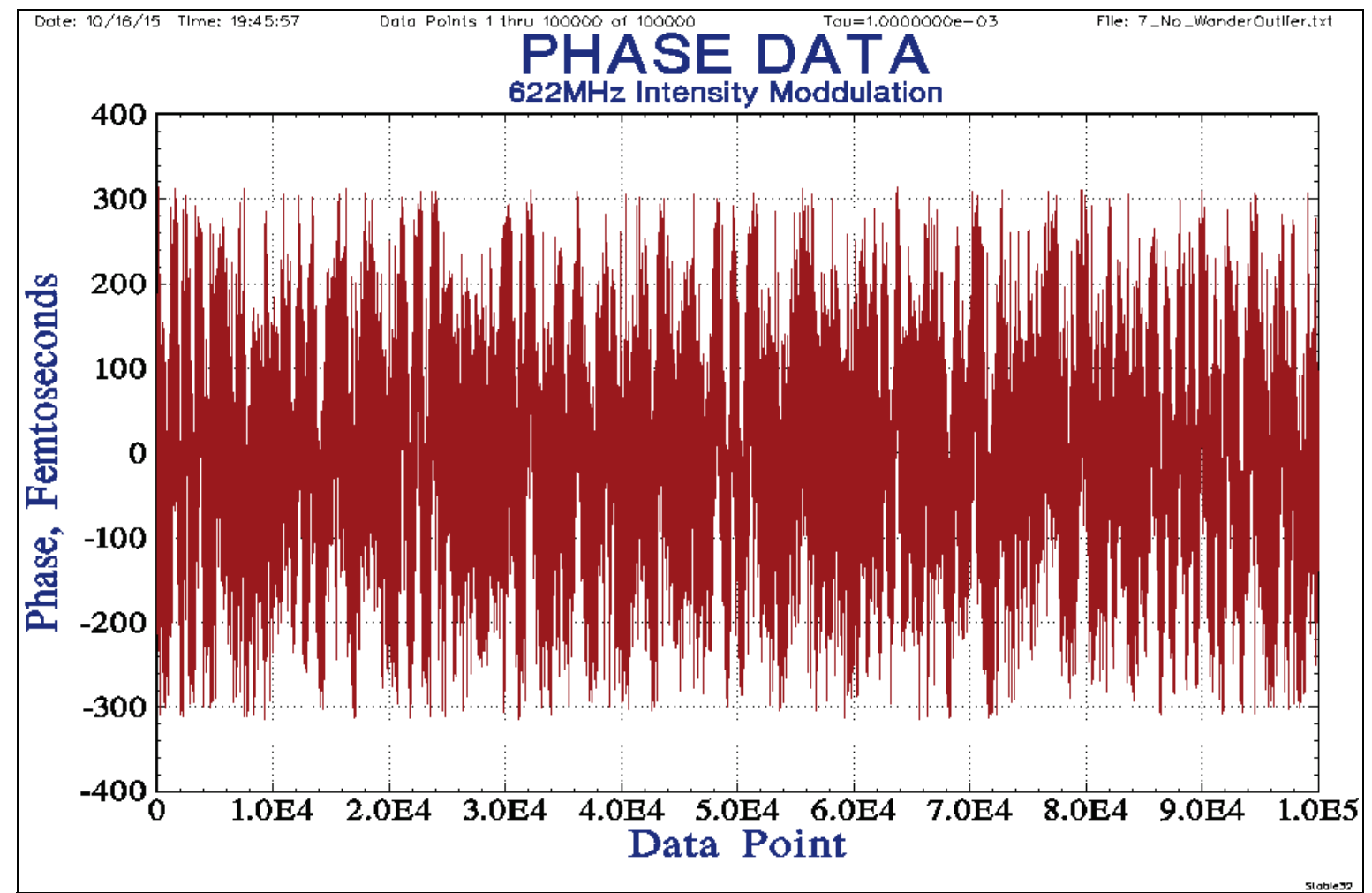

Figure 10. 622 Hz tone amplitude modulation ranging, phase measurements with an averaging time of $0.001 \mathrm{~s}$.

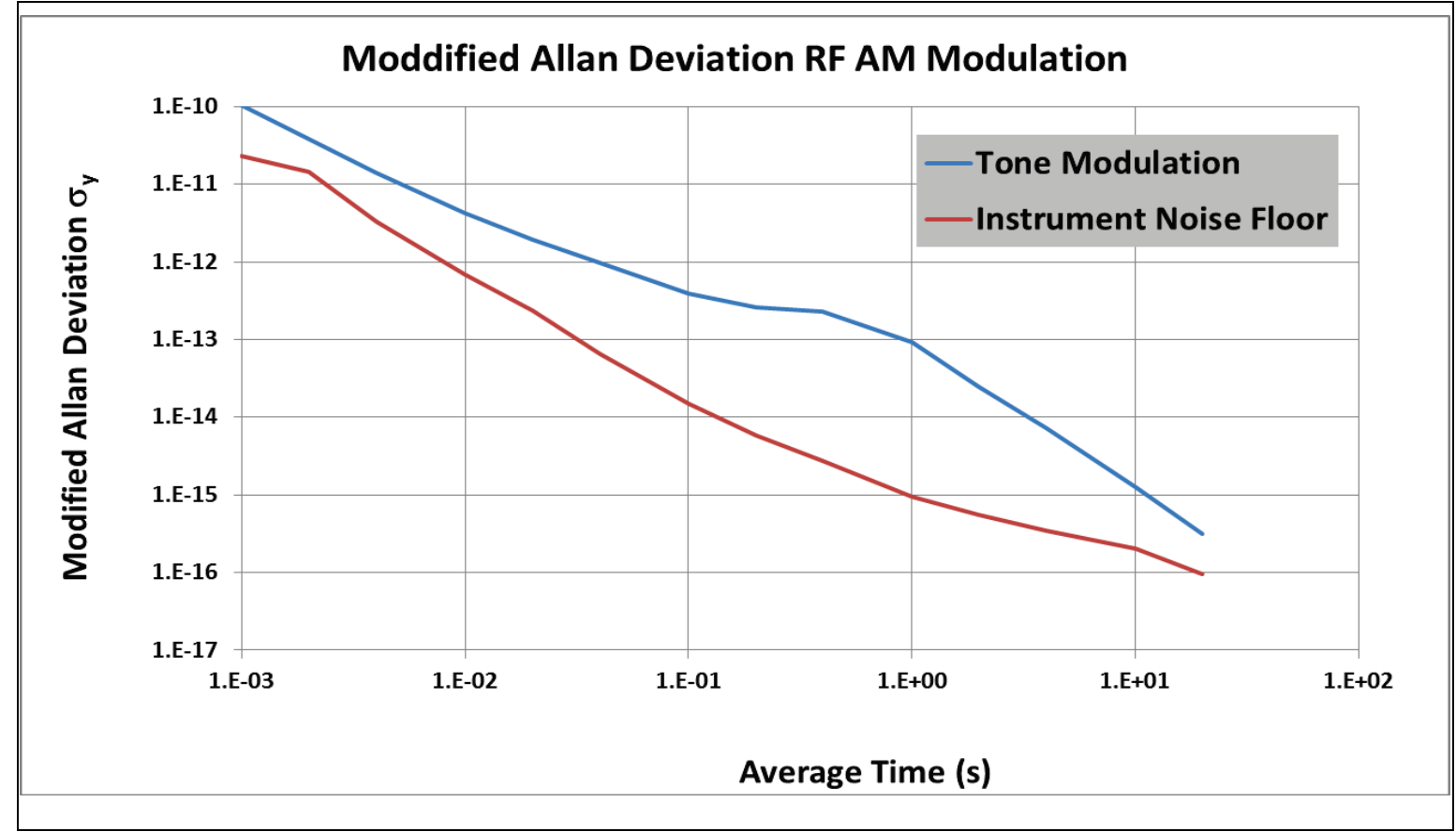

Figure 11. 622MHz tone amplitude modulation ranging Modified Allan deviation and instrument noise floor. 


\section{DISCUSSION}

A DMTD system for accurate clock phase measurements has been built. The system exhibits a range rate $0.28 \mu \mathrm{m} / \mathrm{s}$ for 1 second averaging time and $0.06 \mu \mathrm{m} / \mathrm{s}$ noise floor (see Table 1). The range rate measurements over an optical communication link at $622 \mathrm{Mbps}$ for different optical power level (corresponds to different bit error rate) were tested. A range-rate of 23.1 $\mu \mathrm{m} / \mathrm{s}$ and $2.1 \mu \mathrm{m} / \mathrm{s}$ for 1 second and 10 seconds averaging time respectively are achieved (see Table 1). The range-rate does not change with the received optical power or bit-error-rate (see Fig. 9). This indicates that the shot and thermal noise are not the dominant noise sources. The dominant noise source is electronic noise. This is understandable since all of the optical transmitter and receiver, CDR and corresponding amplifiers are commercial-off-the-shelf parts. To improve the signal-tonoise ratio, single-tone optical amplitude modulation was used. Range-rates of $19.74 \mu \mathrm{m} / \mathrm{s}$ for 1 second averaging time and $0.27 \mu \mathrm{m} / \mathrm{s}$ for 10 seconds averaging time were achieved (see Table 1). Compared to the communication configuration, range-rate results at 10 second averaging time have almost an order of magnitude better performance - improving from 2.1 $\mu \mathrm{m} / \mathrm{s}$ to $0.27 \mu \mathrm{m} / \mathrm{s}$. However, the range-rate at 1 second averaging time improved very little. This is due to the observed flicker FM noise from 0.1 to 1 second averaging time (see Fig. 13). The mostly likely cause for this flicker noise is the commercial OC-12 receiver and the RF mixer for frequency down-converting in the DMTD.

For comparison, the phase-noise power-spectral-density in $\mathrm{dBc} / \mathrm{Hz}$ is calculated from each phase measurement point (see Fig.12, Fig.13, and Fig. 14). We chose a phase noise at $2 \mathrm{~Hz}$ Fourier frequency for comparison (see Table 2). This is the point right before the FM flicker noise in the tone modulation is approaching the maximum (see Fig. 13). The phase noise is $120 \mathrm{dBc} / \mathrm{Hz}$ (instrument), $-90 \mathrm{dBc} / \mathrm{Hz}$ (tone modulation), and $80 \mathrm{dBc} / \mathrm{Hz}$ (communication link).
The noise increase of communication link compared to the tone modulation is mainly due to spectral spreading from the data modulation, which reduced the power level of the 622 $\mathrm{MHz}$ data clock. This is supported with the following test. A test was configured with direct optical loopback at space terminal. So that the optical signal bypasses space receive, transmitter and CDR. Same ranging accuracies were obtained as the fully configured communication link. We are funded to work on theoretical modeling this year. It will enable us to better understand all of the noise sources, performance penalties. The proper modeling will enable us to further improve the overall performance.

The phase noise density increased from $-120 \mathrm{dBc}$ (RF baseline) to $-90 \mathrm{dBc}$ (tone modulation). Two major sources can contribute to this increase. One is the time delay between the DMTD start and stop times introduced by the reference clock timing error $[12,16,17]$, the other source is electronic noise from the optical transmitter, receiver and RF mixer. The electronic noise can be further improved with customer made components.

The phase noise is directly proportional to Allan deviation $[13,14,15]$. There is room for further improvement in both ranging modes phase noise. These improvements will increase the range-rate accuracy beyond the current values.

Table 1. Range rate accuracy

\begin{tabular}{|c|c|c|c||}
\hline \multirow{2}{*}{$\begin{array}{c}\text { Average Time } \\
(\mathrm{s})\end{array}$} & $\begin{array}{c}|c| \\
\text { Instrument } \\
\text { Noise Floor }\end{array}$ & $\begin{array}{c}\text { R22MHz Tone AM } \\
\text { Modulation }\end{array}$ & $\begin{array}{c}\text { 622MBPS Data } \\
\text { Communiation }\end{array}$ \\
\hline 1 & 0.28 & 19.74 & 23.1 \\
\hline 10 & 0.06 & 0.27 & 2.1 \\
\hline \hline
\end{tabular}




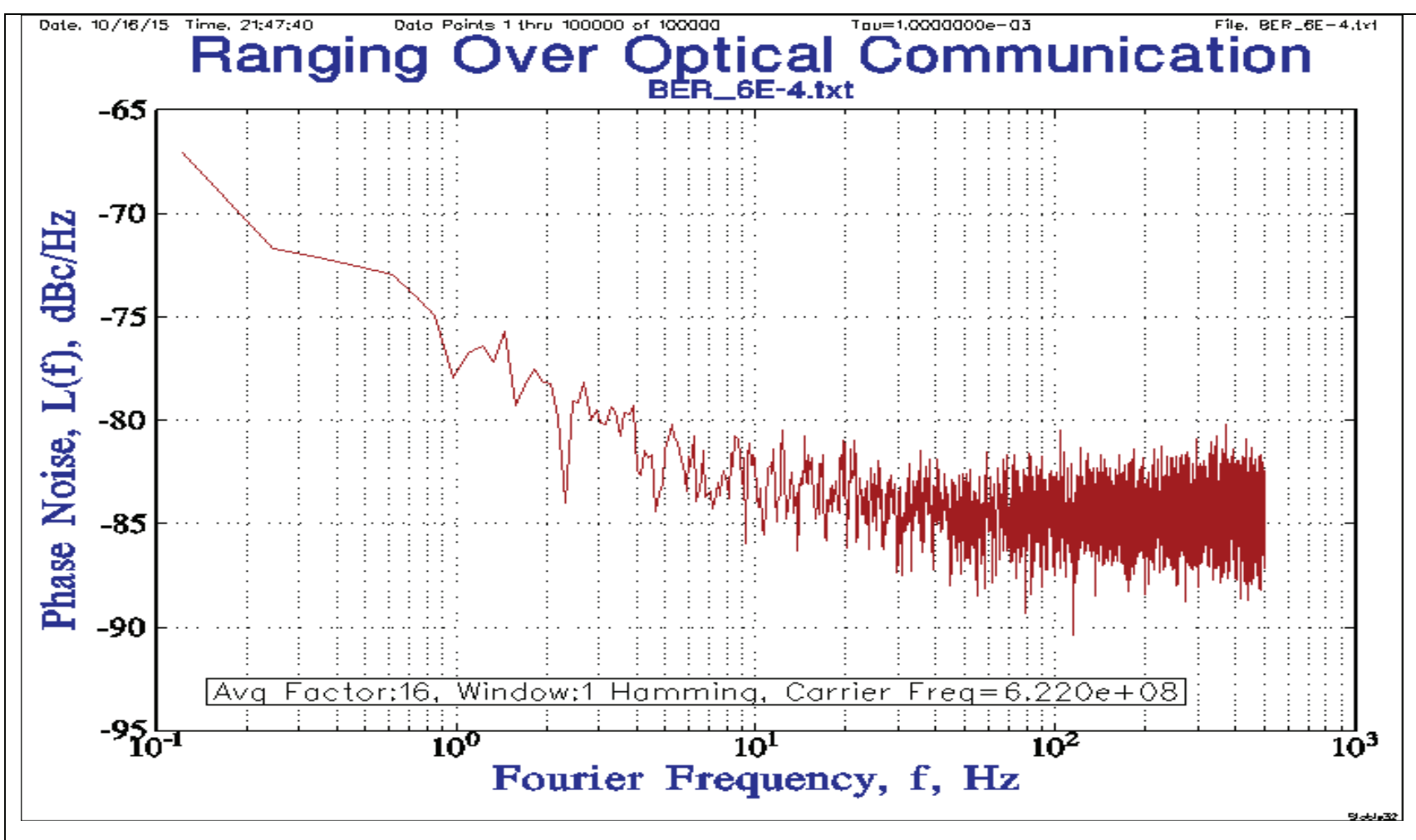

Figure 12. Single side band phase noise power spectral density of optical communication mode.

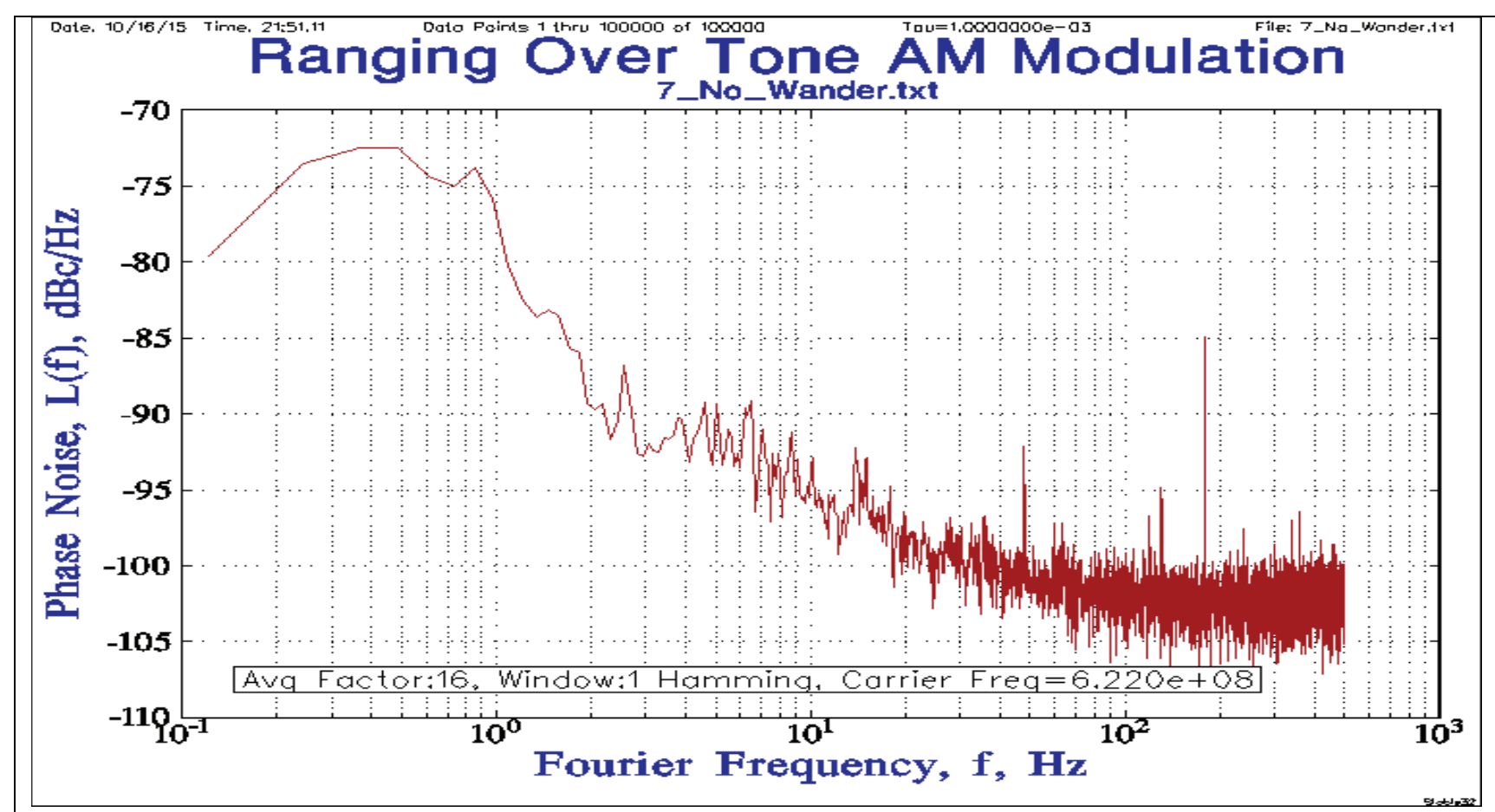

Figure 13. Single side band phase noise power spectral density of Single Tone amplitude Modulation mode. 


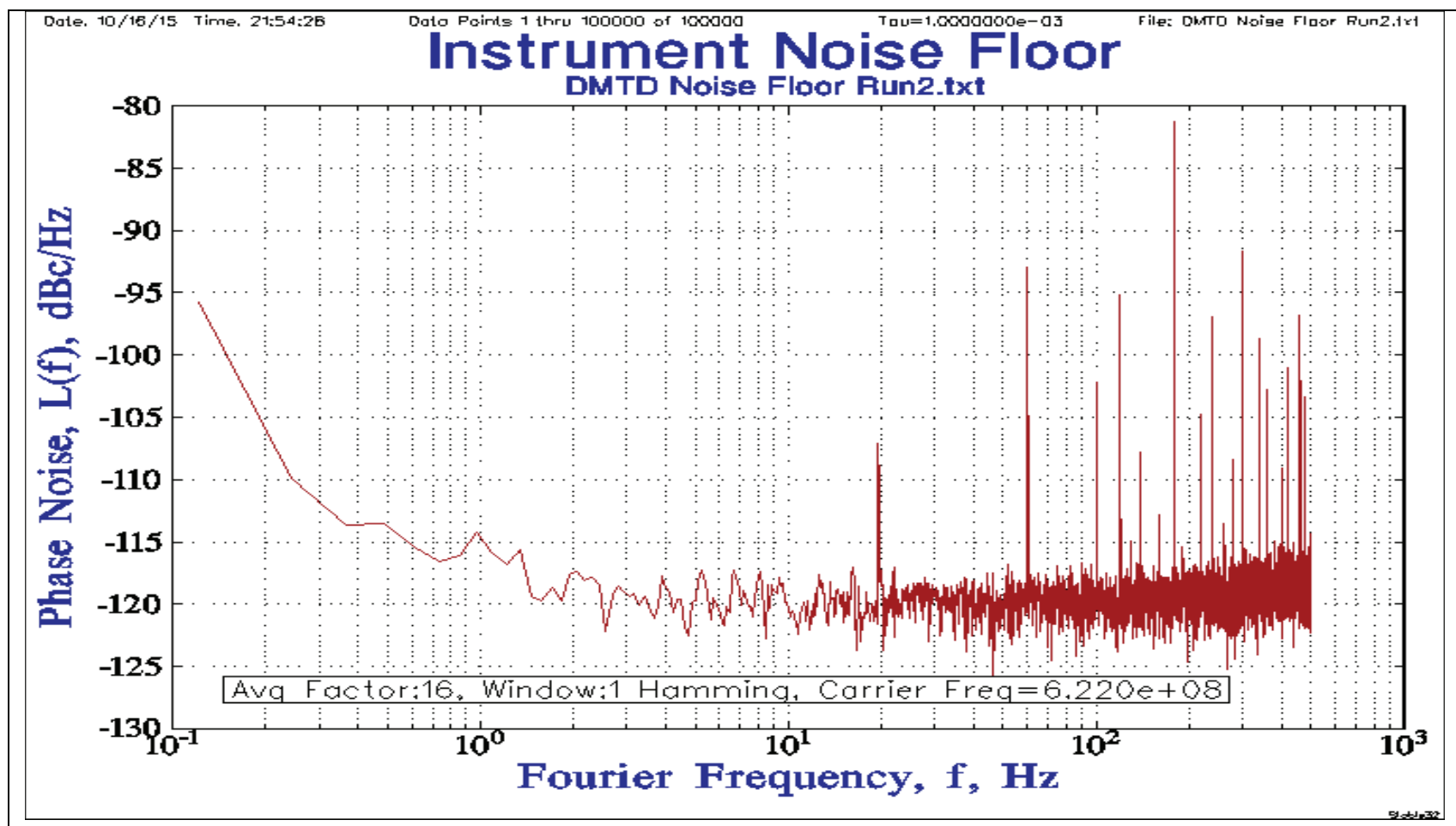

Figure 14. Single side band phase noise power spectral density of Instrument Noise Floor

Table 2. Phase noise power spectra density

\begin{tabular}{|c|c|c|c|}
\hline \multirow{2}{*}{$\begin{array}{c}\text { Fourier } \\
\text { Freequency }(\mathrm{Hz})\end{array}$} & \multicolumn{3}{|c|}{ Phase Noise Power Spectral Density (dBc/Hz) } \\
\cline { 2 - 4 } & $\begin{array}{c}\text { Instrument } \\
\text { Noise Floor }\end{array}$ & $\begin{array}{c}\text { 622MHz Tone AM } \\
\text { Modulation }\end{array}$ & $\begin{array}{c}\text { 622MBPS Data } \\
\text { Communiation }\end{array}$ \\
\hline 2 & -120.00 & -90.00 & -80 \\
\hline \hline
\end{tabular}

\section{CONCLUSION}

A laboratory test bed laser communication link with mock space and ground terminal transmitter and receiver hardware was constructed. The link was used to evaluate the range-rate performance by measuring the phase of the recovered data clock. Range-rate accuracies of $23.1 \mu \mathrm{m} / \mathrm{s}$ and $2.1 \mu \mathrm{m} / \mathrm{s}$ for $1 \mathrm{~s}$ and $10 \mathrm{~s}$ averaging time respectively were achieved. We demonstrated that this range-rate does not change with the $\mathrm{BER}$ rate and received optical power for powers as low as -33 $\mathrm{dBm}$. Improvements of the range-rate accuracy from the communication configuration were tested with a single-tone optical amplitude modulation system at $622 \mathrm{MHz}$. Range-rate accuracies of $19.7 \mu \mathrm{m} / \mathrm{s}$ and $0.27 \mu \mathrm{m} / \mathrm{s}$ for $1 \mathrm{~s}$ and $10 \mathrm{~s}$ averaging time respectively were achieved. All phase measurements are performed with a DMTD test apparatus which has a measured noise floor of $0.28 \mu \mathrm{m} / \mathrm{s}$ and $0.06 \mu \mathrm{m} / \mathrm{s}$ for $1 \mathrm{~s}$ and $10 \mathrm{~s}$ averaging time respectively. Both experimental ranging mode performance was limited by the use of commercial-off-the-shelf components from the telecommunication OC-12 family. We are building an improved version of the hardware using custom components to address this.

The achieved range-rate accuracy has significant importance for both space navigation and communication. It enables an almost no-cost, very high-precision ranging service for any free-space optical communication system. This is because the range measurement is conducted with the recovered data clock, which is required in all synchronized optical communication systems. Another important application for our work is high-precision range measurements in science applications, (e.g. gravity-wave measurements of GRAIL and GRACE). These systems use RF signal tones for ranging, where plasma scintillation is a major performance limitation [1]. A special design is required to correct plasma introduced phase noise[5]. Further, it remains a major error source when the Sun-Earth-Probe (SEP) angle is less than 30 degrees [5]. The plasma-induced refractive-index fluctuation is proportional to the square of carrier wavelength [5]. The optical carrier is 4 orders of magnitude shorter wavelength than $10 \mathrm{GHz} \mathrm{RF}$ carrier. The short optical wavelength eliminates the plasma induced noise issue for optical ranging. High-precision optical ranging has another intrinsic advantage due to the short wavelength. It has very high antenna gain (small beam divergence) compare to RF. This small beam divergence (high antenna gain) can be used in two ways: to decrease the size of the ranging equipment, and/or to increase the ranging distance. This makes optical ranging instruments attractive for deepspace small satellite and cubesat missions. Optical communication ranging provides precise absolute range measurements due to the synchronized operation[3], it provides 
another dimension of measurement for science missions to explore.

\section{ACKNOWLEDGMENT}

This work is funded by NASA GODDARD CIF/IRAD fund CIF295670

The authors would like to thank Dr. Jeffery Chen of NASA Goddard Flight Space Center for many valuable discussions and recommendations on both theoretical and experimental aspects of the work.

\section{REFERENCES}

[1] S. W. Asmar, J. W. Armstrong, "Spacecraft Doppler tracking: Noise budget and accuracy achievable in precision radio science observations," Radio Science, VOL. 40, RS2001, doi:10.1029/2004RS003101, 2005

[2] Iess, L., Dobrowolny, M., Bertotti B., 'Plasma effects on Doppler measurements of interplanetary spacecraft', Astronomy and Astrophysics, 121, 203-210, 1983

[3] Boroson, D.M. et al. "The Lunar Laser Communications Demonstration (LLCD)." Third IEEE International Conference on Space Mission Challenges for Information Technology.

[4] Maria T. Zuber · David E. Smith, et al. "Gravity Recovery and Interior Laboratory (GRAIL): Mapping the Lunar Interior from Crust to Core" Space Science Review, DOI 10.1007/s11214012-9952-7

[5] Luciano Iess, Mauro Di Benedetto, et al. "Improved Doppler Tracking System for Deep Space Navigation" International Symposium on Space Flight Dynamics (ISSFD), 2012, ISSFD23_OD1_6.

[6] University of ROMA LA SAPIENZA, "Interdisciplinary Study on Enhancement of End-To-End Accuracy for Spacecraft Tracking Techniques",ESA, General Study report, 14 March 2012.

[7] B. D. Tapley, S. Bettadpur, et al. "The Gravity Recovery and Climate Experiment: Mission Overview and Early Results" GEOPHYSICAL RESEARCH LETTERS, VOL. 31, L09607, doi:10.1029/2004GL019920, 2004.

[8] David E. Smith, Maria T. Zuber, et al. "Science and High Accuracy Laser Ranging between the Planets", Dave Smith's EGU talk, 2015

[9] Iess, L., Dobrowolny, M., Bertotti B., "Plasma effects on Doppler measurements of interplanetary spacecraft", Astronomy and Astrophysics, 121, 203-210, 1983

[10] W.J. Riley, "Handbook of Frequency Stability Analysis", NIST Special Publication 1065, pp 81.

[11] Samuel R. Stein, 1985, "Frequency and Time - Their Measurement and Characterization," Precision Frequency Control, Vol. 2, Chap. 12, pp. 203-204

[12] W.J. Riley, "A Small Dual Mixer Time Difference (DMTD) Clock Measuring System," Manual, Hamilton Technical Services Beaufort, SC 29907 USA

[13] D. Allan, H. Hellwig, P. Kartaschoff, J. Vanier, J. Vig, G. M. R. Winkler, and N. Yannoni, 1988,

[14] "Standard Terminology for Fundamental Frequency and Time Metrology," in Proceedings of the 42nd Annual Symposium on Frequency Control, 1-3 June 1988, Baltimore, Maryland, USA (IEEE 88CH2588-2), pp. 419-425.
[15] P. C. Chang, H. M. Peng, and S. Y. Lin, "ALLAN VARIANCE ESTIMATED BY Phase NOISE MEASUREMENTS," 36th Annual Precise Time and Time Interval (PTTI) Meeting

[16] C. A. Greenhall, "Common-Source Phase Noise of a DualMixer Stability Analyzer", TMO Progress Report 42-143, Jet Propulsion Laboratory, Pasadena, California, USA, November 2000.

[17] C.A. Greenhall, A. Kirk, and G.L. Stevens, "A Multichannel Dual-Mixer Stability Analyzer: Progress Report", Proceedings of the 33th Precise Time and Time Interval (PTTI) Applications and Planning Meeting, pp. 377-384, November 2001.

\section{BIOGRAPHY}

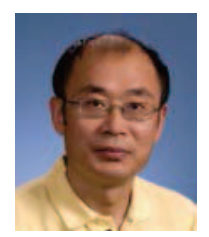

Guangning Yang received his BS in physics from Sichuan University, Chengdu, China and $P h D$ in Electrical Engineering from Drexel University. He worked at Tyco Submarine Systems as lead design engineer for the first $10 G B P S$ transmitter for trans-oceanic optical communication. In 2000, he worked as the Technical Section Lead for high speed optical communication terminal for Dorsal/Corvis Networks. For the past eight years he has worked at NASA Goddard Space Flight Center (GSFC) on lidar and laser ranging and communication. He is the Product Development Lead on photon counting detector for the ATLAS Laser Altimeter instrument on ICESAT-2 Mission.

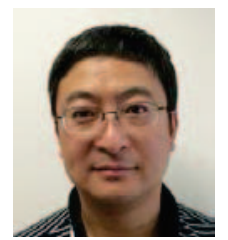

Wei $\mathbf{L u}\left(M^{\prime} 95\right)$ received the B.S. degree from Shanghai University of Technologies, China, in 1989, and the MS. degree from Shanghai Institute of Technical Physics, Chinese Academy of Sciences in 1996, both in Electrical Engineering. He started his career as Associated Research on infrared focal plan array and signal processing at SITP and have earned several national awards for his achievement. Later, he design and manufactured many small form factor optical transceivers in Optical Communications Products, Inc. He is now a Senior Staff Engineer of ASRC Federal Inc at NASA's Goddard Space Flight Center, Greenbelt, MD, USA. He is the lead engineer in photon counting detector development for Advanced Topographic Laser Altimeter System (ATLAS) ICESAT-2 Mission. His primary focus is detector technologies, optical communication and ranging, electronic circuit design. He has been actively volunteering in the local community in his home state of Delaware. For years, he teams up University of Delaware Electrical and Computer Engineering Department to teach middle and high school students all over the State on the basic theory of electricity and circuits.

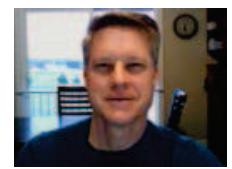

Michael Krainak received his $B S$ in electrical engineering from Catholic University and $M S$ and $P h D$ in Electrical Engineering from Johns Hopkins University. He started his career as a telephone switch office field 
engineer for AT\&T Western Electric. He worked for ten years at the National Security Agency in signal processing, Fourier optics, and microelectronic circuit design. In 2001, he worked as the Test and Reliability Manager at the semiconductor laser manufacturer start-up - Quantum Photonics Inc. For the past twenty-four years he has worked at NASA Goddard Space Flight Center (GSFC) on inter-satellite laser communications and lidar. Dr. Krainak is the Head of the Laser and ElectroOptics Branch at NASA-GSFC.

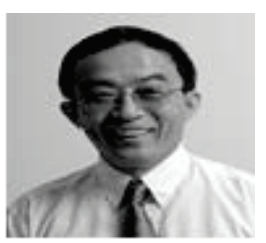

Xiaoli Sun (S'88-M'90) received the B.S. degree from Taiyuan Institute of Technology, Taiyuan, China, in 1982, and the Ph.D. degree in electrical engineering from the Johns Hopkins University, Baltimore, MD, USA, in 1989. He is now a Research Scientist in the Solar System Exploration Division at the NASA Goddard Space Flight Center, Greenbelt, MD, USA. He was the Lead Engineer in photodetector development and receiver performance analysis for the Mars Orbiter Laser Altimeter on the Mars Global Surveyor Mission and the Geoscience Laser Altimeter System on the ICESat Mission. He is the instrument scientist for the Mercury Laser Altimeter on the MESSENGER mission and the Lunar Orbiter Laser Altimeter and the one-way Laser Ranging subsystem on the Lunar Reconnaissance Orbiter (LRO) mission. He led the first two-way laser ranging experiment between the MESSENGER spacecraft and Earth over 24 million $\mathrm{km}$ in 2005, and the first lunar laser communication experiments from Earth to the laser altimeter on the Lunar Reconnaissance Orbiter in 2012. He is the lead research scientist for infrared lidar detector development and receiver modeling for trace gas lidar, such as the CO2 lidar for NASA's Active Sensing of CO2 Emission over Nights, Days, and Seasons (ASCENDS) mission. 